\title{
Impacto de las revistas de psiquiatría y salud mental editadas en español.
}

\author{
Impact of psychiatry and mental health journals published in Spanish. \\ Johann M. Vega-Dienstmaier ${ }^{1, a}$ \\ Identificación ORCID: https://orcid.org/0000-0002-5686-4014
}

\section{RESUMEN}

Objetivo: Describir el impacto de las revistas sobre psiquiatría y salud mental editadas en español. Material y Métodos: Se identificaron las revistas sobre psiquiatría y salud mental publicadas en castellano, incluidas en Scopus, Google Académico y Scielo. Cada revista fue evaluada en base a los siguientes indicadores de impacto: CiteScore mejorado (Scopus); índice h5 y mediana h5 (Google Académico); y factor de impacto basado en 3 años e índice de inmediatez (SciELO). Resultados: Las revistas con mayor impacto fueron de España (v.g. Psicothema y Revista de Psiquiatría y Salud Mental), Colombia (v.g. Revista Latinoamericana de Psicología y Revista Colombiana de Psiquiatría), Chile (v.g. Terapia Psicológica y Psicoperspectivas) y México (v.g. Salud Mental y Revista Mexicana de Psicología). En Perú las revistas más relevantes fueron Revista de Psicología y Revista de Neuro-Psiquiatría. Conclusiones: Dentro de las revistas internacionales de psiquiatría y salud mental que aceptan artículos en español, las de mayor impacto fueron publicaciones de España, con Psicothema, como la más destacada.

PALABRAS CLAVE: Bibliometría, factor de impacto, revistas, publicaciones periódicas.

\section{SUMMARY}

Objective: To describe the impact of psychiatry and mental health journals published in Spanish. Material and Methods: The psychiatry and mental health journals published in Spanish and included in Scielo, Scopus and Google Scholar were identified. The following impact indicators were obtained for each journal: improved CiteScore (Scopus); h5 index and h5 median (Google Scholar); and impact factor based on 3 years and immediacy index (Scielo). Results: The journals with the greatest impact were from Spain (eg, Psicothema and Revista de Psiquiatría y Salud Mental), Colombia (eg, Revista Latinoamericana de Psicología and Revista Colombiana de Psiquiatría), Chile (eg, Terapia Psicológica and Psicoperspectivas), and Mexico (eg, Salud Mental and Revista Mexicana de Psicología). In Peru the most relevant journals were Revista de Psicología and Revista de Neuro-Psiquiatría.

Conclusions: Among the international journals of psychiatry and mental health that accept articles in Spanish, the highest impact indicators were found in publications from Spain, with Psicothema outstanding.

KEYWORDS: Bibliometrics, impact factor, journals, periodicals.

\footnotetext{
1 Universidad Peruana Cayetano Heredia, Facultad de Medicina Alberto Hurtado.Lima, Perú.

a Médico-psiquiatra; Magister en epidemiología clínica.
} 
Impacto de las revistas de psiquiatría y salud mental editadas en español.

\section{INTRODUCCIÓN}

El impacto de una revista suele medirse considerando la cantidad de veces que sus artículos son citados en las publicaciones incluidas en las bases de datos de literatura científica tal como lo hacen Scopus y Web of Science (1). Para este fin se han desarrollado varios indicadores tales como el factor de impacto, el CiteScore, el SCImago Journal Rank y el índice h (2).

El número de artículos científicos sobre salud mental publicados por autores de instituciones peruanas en revistas indexadas es bajo de acuerdo a información analizada procedente de Scopus entre 1992 y 2018 (3), y entre 2011 y 2013 (4); y de SCImago entre 1996 y 2018 (5). Asimismo, una buena parte de estas revistas abarcan temas más generales y no son específicamente sobre salud mental, entre ellas tenemos la Revista Peruana de Medicina Experimental y Salud Pública (3-5), Plos One (3,5), AIDS and Behavior (5), Lancet (5) y la Revista de Gastroenterología del Perú (5).

Conocer las diversas revistas que aceptan artículos en castellano y tener información sobre su calidad y desempeño es relevante para los autores de Perú y Latinoamérica porque facilitaría la publicación de sus trabajos en los medios de difusión científica más convenientes. Asimismo, dicha información sería de interés para los editores y cuerpos editoriales de las revistas en español sobre psiquiatría y salud mental.

El objetivo del presente estudio fue identificar las revistas sobre salud mental que se publican en español en diferentes partes del mundo y describir su impacto.

\section{MATERIAL Y MÉTODOS}

El presente trabajo es un estudio descriptivo bibliométrico.

Durante el mes de septiembre del 2021, se identificaron las revistas relacionadas con salud mental en Scielo, Scopus y Google Académico, de la siguiente manera:

- En Scielo: se descargó el archivo de la lista de sus 1831 revistas con sus respectivos datos (6); en base a esta lista se ubicaron las publicaciones cuyo nombre o sus datos tenían las palabras "psicología", "psiquiatría”, "mental", "neuropsiquiatría", "psicológica", "psicológico", "psiquiátrico" y "psiquiátrica".

- En Google Académico: se accedió a la sección "Estadísticas" y se utilizó el motor de búsqueda de este sitio para ubicar las publicaciones correspondientes a las palabras clave "psicología", "psiquiatría", "mental”, "psicológica", "psicológico", "psiquiátrico", "psiquiátrica” y "neuropsiquiatría" (7).

- En Scopus: se revisaron las revistas dentro de la categoría "Psychiatry and Mental Health". La existencia de esta categoría en Scopus facilitó la identificación de las revistas relacionadas con los objetivos del estudio, no siendo necesario la utilización de palabras clave con la finalidad de ubicar las publicaciones sobre psiquiatría y salud mental como se hizo en los dos casos previos. La categoría "Psychiatry and Mental Health" incluye a 794 revistas publicadas en diversos idiomas.

En los 3 casos, sólo se incluyeron las revistas que recibían artículos escritos en español (aunque también era posible que acepten además artículos en otros idiomas como el inglés o portugués), se revisó el link de cada revista para verificar si realmente correspondía a una publicación sobre salud mental y se identificó el país donde se edita. Se excluyeron las revistas que al momento del estudio no aceptaban artículos escritos en español (aunque lo hubieran hecho antes en algún momento de la historia de la publicación).

Para cada revista identificada se buscaron los siguientes indicadores de impacto:

- CiteScore mejorado 2020 de Scopus: contabiliza las citas recibidas entre el 2017 y 2020 de publicaciones realizadas entre 2017 y 2020 y las divide entre el número de publicaciones realizadas entre 2017 y 2020 (8). Este indicador difiere ligeramente del CiteScore previo (2).

- Índice h5 de Google Académico: índice h para los artículos publicados en los últimos 5 años completos, en otras palabras, el máximo número $\mathrm{h}$ tal que $\mathrm{h}$ artículos publicados entre 2016 y 2020 tengan al menos $\mathrm{h}$ citaciones cada uno (9).

- La mediana h5 de Google Académico: mediana del número de citas de los artículos que conforman el índice h5 (9).

- Factor de impacto según Scielo del 2020 basado en 3 años: número de citas en el 2020 de los artículos publicados en el 2017, 2018 y 2019 dividido entre número de artículos publicados en el 2017, 2018 y 2019 (2).

- Índice de inmediatez 2020 de Scielo: número de citas en el 2020 a artículos publicados en el 2020 dividido entre la cantidad de artículos publicados en el 2020 (10). 
Para cada país, se calculó el número total de revistas identificadas mediante al menos alguna de las 3 fuentes: Scopus, Google Académico y Scielo; y la cantidad de revistas que estaban activas de acuerdo a cada fuente, es decir, que estaban actualizadas y con sus indicadores bibliométricos disponibles.

El presente trabajo, al ser de tipo bibliométrico y por lo tanto no incluir como objetos de estudio seres humanos o animales, no requirió ser revisado y aprobado por un comité de ética.

\section{RESULTADOS}

En la tablas 1, 2 y 3 se listan las revistas con sus respectivos países y sus indicadores de impacto. La tabla 1 muestra las revistas incluidas en Scopus ordenadas de acuerdo a su CiteScore mejorado; la

Tabla 1. Indicadores de impacto de las revistas editadas en español incluidas en Scopus.

\begin{tabular}{|c|c|c|c|c|c|c|}
\hline Revista & País & CS & h5 & h5m & FIS & IIS \\
\hline Psicothema & España & 5,3 & 35 & 54 & - & - \\
\hline Adicciones & España & 4,8 & 25 & 36 & - & - \\
\hline Revista de Psiquiatría y Salud Mental & España & 3,6 & 21 & 28 & - & - \\
\hline Anales de Psicología & España & 3,3 & 31 & 51 & 0,3146 & 0,0500 \\
\hline $\begin{array}{l}\text { Revista de Psicología del Trabajo y de las } \\
\text { Organizaciones }\end{array}$ & España & 3,1 & 28 & 49 & 0,0725 & 0,0000 \\
\hline Revista Latinoamericana de Psicología & Colombia & 2,4 & 20 & 39 & - & - \\
\hline Revista de Psicología del Deporte & España & 2,1 & 24 & 31 & - & - \\
\hline Actas Españolas de Psiquiatría & España & 2,0 & 16 & 17 & - & - \\
\hline Papeles del Psicólogo & España & 1,9 & 24 & 38 & * & 0,0000 \\
\hline Suma Psicológica & Colombia & 1,8 & 18 & 32 & 0,5417 & 0,0625 \\
\hline $\begin{array}{l}\text { Revista Iberoamericana de Psicología del Ejercicio y el } \\
\text { Deporte }\end{array}$ & España & 1,5 & 20 & 33 & - & - \\
\hline Revista de Psicopatología y Psicología Clínica & España & 1,5 & 14 & 30 & - & - \\
\hline Cuadernos de Psicología del Deporte & España & 1,5 & - & - & * & 0,1600 \\
\hline Revista Colombiana de Psicología & Colombia & 1,4 & 19 & 30 & * & $*$ \\
\hline Terapia Psicológica & Chile & 1,2 & 19 & 29 & 0,4627 & 0,0833 \\
\hline Psicoperspectivas (Valparaíso, En línea) & Chile & 1,0 & 21 & 33 & - & - \\
\hline Salud mental & México & 1,0 & 15 & 23 & 0,1837 & 0,0000 \\
\hline Revista Mexicana de Psicología & México & 1,0 & - & - & - & - \\
\hline Clínica y Salud & España & 0,9 & 29 & 45 & 0,3000 & 0,3571 \\
\hline Revista Colombiana de Psiquiatría & Colombia & 0,9 & 20 & 30 & - & - \\
\hline Anuario de Psicología & España & 0,9 & 8 & 16 & - & - \\
\hline Acta Colombiana de Psicología & Colombia & 0,8 & 19 & 33 & 0,3243 & 0.2333 \\
\hline Revista de Psicología (Lima) & Perú & 0,7 & 19 & 32 & 0,0282 & 0,0000 \\
\hline Avances en Psicología Latinoamericana & Colombia & 0,6 & 21 & 29 & 0,4237 & 0,0513 \\
\hline Revista de Psicología Clínica con Niños y Adolescentes & España & 0,5 & 19 & 26 & - & - \\
\hline Interamerican Journal of Psychology & Brasil & 0,5 & 14 & 25 & - & - \\
\hline Psicologia Escolar e Educacional & Brasil & 0,4 & 22 & 30 & - & - \\
\hline Acta Comportamentalia & México & 0,4 & 10 & 16 & - & - \\
\hline CES Psicología & Colombia & 0,3 & 16 & 27 & 0,0441 & 0,0500 \\
\hline Revista Chilena de Neuro-Psiquiatría & Chile & 0,3 & 13 & 27 & 0,0795 & 0,0000 \\
\hline Psiquiatría Biológica & España & 0,2 & 7 & 11 & - & - \\
\hline Neurología, Neurocirugía y Psiquiatría & México & 0,1 & 4 & 5 & - & - \\
\hline Revista de Psicología de la Salud & España & 0,1 & - & - & - & - \\
\hline
\end{tabular}

$\mathrm{CS}=$ CiteScore mejorado (Scopus), h5=índice h5 de Google Académico 2020, h5m=mediana h5 de Google Académico, FIS=Factor de impacto 2020 de Scielo basado en 3 años, IIS=índice de inmediatez 2020 de Scielo.

* Actualizada en Scielo pero sin el indicador de impacto disponible en esta base de datos al momento de realizar el estudio 
Impacto de las revistas de psiquiatría y salud mental editadas en español.

Tabla 2. Indicadores de impacto de las revistas editadas en español no incluidas Scopus pero sí en Google Académico.

\begin{tabular}{|c|c|c|c|c|c|}
\hline Revista & País & h5 & h5m & FIS & IIS \\
\hline Pensamiento Psicológico & Colombia & 22 & 34 & 0,4746 & 0,0000 \\
\hline Temas en Psicología & Brasil & 22 & 31 & - & - \\
\hline $\begin{array}{l}\text { Revista INFAD de Psicología, International Journal of } \\
\text { Developmental and Educational Psychology }\end{array}$ & España & 21 & 33 & - & - \\
\hline Psicología desde el Caribe & Colombia & 20 & 23 & - & - \\
\hline Acta de Investigación Psicológica & México & 17 & 24 & - & - \\
\hline Acción Psicológica & España & 16 & 25 & - & - \\
\hline Revista de Neuro-Psiquiatría & Perú & 15 & 35 & 0,2174 & 0,2059 \\
\hline Actualidades en Psicología & Costa Rica & 13 & 20 & $*$ & $*$ \\
\hline Informes Psicológicos & Colombia & 13 & 26 & - & - \\
\hline Ciencias Psicológicas & Uruguay & 12 & 20 & $*$ & $*$ \\
\hline Psicología, Conocimiento y Sociedad & Uruguay & 12 & 17 & - & - \\
\hline Revista de Psicología (Santiago) & Chile & 11 & 23 & 0,1139 & 0,0000 \\
\hline Revista Puertorriqueña de Psicología & Puerto Rico & 11 & 17 & - & - \\
\hline Revista de la Asociación Española de Neuropsiquiatría & España & 10 & 12 & 0,2564 & 0,0667 \\
\hline Cuadernos de Neuropsicología & Chile & 10 & 16 & - & - \\
\hline Revista Costarricense de Psicología & Costa Rica & 10 & 17 & - & - \\
\hline Límite (Arica) & Chile & 9 & 14 & 0,0000 & 0,0000 \\
\hline Anuario de investigaciones & Argentina & 8 & 8 & - & - \\
\hline Revista de Psicología Universidad de Antioquia & Colombia & 8 & 15 & - & - \\
\hline $\begin{array}{l}\text { Revista de psicología y ciencias del comportamiento de la Unidad } \\
\text { Académica de Ciencias Jurídicas y Sociales }\end{array}$ & México & 7 & 17 & - & - \\
\hline Tesis Psicológica & Colombia & 7 & 10 & - & - \\
\hline Revista del Hospital Psiquiátrico de La Habana & Cuba & 5 & 9 & - & - \\
\hline Norte de Salud Mental & España & 4 & 5 & - & - \\
\hline Psicosomática y Psiquiatría & España & 4 & 6 & - & - \\
\hline $\begin{array}{l}\text { Informaciones psiquiátricas: Publicación científica de los Centros } \\
\text { de la Congregación de Hermanas Hospitalarias del Sagrado } \\
\text { Corazón de Jesús }\end{array}$ & España & 3 & 5 & - & - \\
\hline Vertex: Revista Argentina de Psiquiatría & Argentina & 3 & 4 & - & - \\
\hline $\begin{array}{l}\text { Cuadernos de Psiquiatría y Psicoterapia del Niño y del } \\
\text { Adolescente }\end{array}$ & España & 2 & 7 & - & - \\
\hline
\end{tabular}

h5=índice h5 de Google Académico 2020, h5m=mediana h5 de Google Académico, FIS=Factor de impacto 2020 de Scielo basado en 3 años, IIS=índice de inmediatez 2020 de Scielo.

* Actualizada en Scielo pero sin el indicador de impacto disponible en esta base de datos al momento de realizar el estudio.

tabla 2, las que no están en Scopus pero sí en Google Académico ordenadas según su índice h5; y la tabla 3, las que sólo están en Scielo ordenadas en base a su factor de impacto y si éste indicador no se reporta, primero aparecen las que están al día y por último las que no tienen sus números actualizados.

La tabla 4 indica la cantidad de revistas por país: en total y las indexadas activas en Scopus, Google Académico y Scielo; evidenciándose que los países con mayor número de revistas, en total o indexadas, fueron España y Colombia.

\section{DISCUSIÓN}

Considerando las revistas sobre salud mental publicadas en español, Psicothema es la que tiene los más altos indicadores de impacto de Scopus (CiteScore) y Google Académico (índice h5) (11); esta revista española, fue fundada en 1989 y es editada por la Universidad de Oviedo y el Colegio Oficial de Psicólogos del Principado de Asturias; asimismo, es de acceso abierto y está indexada a múltiples bases de datos. Psicothema ha sido estudiada bibliométricamente de forma individual (12), y en 
Tabla 3. Indicadores de impacto de las revistas editadas en español no incluidas en Scopus ni Google Académico pero sí en Scielo.

\begin{tabular}{lccc}
\hline Revista & País & FIS & IIS \\
\hline Liberabit & Perú & 0,2545 & 0,0000 \\
Psicumex & México & 0,0000 & 0,0000 \\
Ajayu: Órgano de Difusión Científica del Departamento de Psicología UCBSP & Bolivia & $*$ & $*$ \\
Escritos de Psicología (Internet) & España & $*$ & $*$ \\
Revista de Investigación Psicológica & Bolivia & $*$ & $*$ \\
Revista Psicodebate: Psicología, Cultura y Sociedad. & Argentina & $*$ & $*$ \\
Alternativas en Psicología & México & - & - \\
Boletín (Sociedad de Psicología del Uruguay, en línea) & Uruguay & - & - \\
Orientación y Sociedad & Argentina & - & - \\
Persona (Lima) & Perú & - & - \\
Revista Cubana de Psicología & Cuba & - & - \\
Revista de Investigación en Psicología & Perú & - & - \\
Revista de Psicología - Universidad Central de Venezuela & Venezuela & - & - \\
Revista Electrónica de Psicología Política & Argentina & - & - \\
Summa Psicológica UST (en línea) & Chile & - & - \\
\hline
\end{tabular}

FIS=Factor de impacto 2020 de Scielo basado en 3 años, IIS=índice de inmediatez 2020 de Scielo.

* Actualizada en Scielo pero sin el indicador de impacto disponible en esta base de datos al momento de realizar el estudio.

Tabla 4. Número de revistas por país en total y en actividad* indexadas en cada una de las 3 fuentes.

\begin{tabular}{lcccc}
\hline País & Total & Scopus* & Google* $^{*}$ & Scielo* $^{*}$ \\
\hline España & 24 & 16 & 21 & 4 \\
Colombia & 12 & 7 & 12 & 5 \\
México & 8 & 4 & 5 & 2 \\
Chile & 7 & 3 & 6 & 4 \\
Perú & 5 & 1 & 2 & 3 \\
Argentina & 5 & 0 & 2 & 0 \\
Brasil & 3 & 2 & 3 & 0 \\
Uruguay & 3 & 0 & 2 & 0 \\
Costa Rica & 2 & 0 & 2 & 0 \\
Cuba & 2 & 0 & 1 & 0 \\
Bolivia & 2 & 0 & 0 & 0 \\
Puerto Rico & 1 & 0 & 1 & 0 \\
Venezuela & 1 & 0 & 0 & 0 \\
Total & 75 & 33 & 57 & 18 \\
\hline * No se contabilizaron las revistas que no estaban al día y que la fuente no \\
reportaba los correspondientes indicadores bibliométricos.
\end{tabular}

comparación con otras revistas (13); además, tiene el mayor índice de internacionalidad de las revistas iberoamericanas de psicología incluidas en Web of Science (14). El índice de internacionalidad de la revista toma en cuenta el idioma de publicación (mayor puntaje si es en inglés), el acceso por Internet (mayor puntaje si hay disponibilidad del artículo a texto completo y si éste es gratuito), si se adecúa a las normas internacionales de publicación, la inclusión en diversas bases de datos, el factor de impacto, la diversidad de países de los que proceden los miembros del comité editorial y los autores; y finalmente, si la institución a la que pertenece o el nombre de la misma revista tiene la palabra "internacional" (14). El impacto de Psicothema podría deberse al hecho de publicar principalmente en inglés y ser de acceso abierto. 
Impacto de las revistas de psiquiatría y salud mental editadas en español.

España y Colombia son los países que más destacan en cuanto a las publicaciones sobre salud mental en castellano teniendo el mayor número de revistas en total o indexadas a Scopus, Google Académico y Scielo.

Las publicaciones que tienen mayor impacto son de España, entre ellas sobresalen por su CiteScore (CS): Psicothema (CS=5,3), Adicciones $(\mathrm{CS}=4,8)$ y la Revista de Psiquiatría y Salud Mental (CS=3,6) (15); y por sus mayores índices h5: Psicothema (h5=35), Anales de Psicología (h5=31) (16) y Clínica y Salud (h5=29) (17).

Considerando el factor de impacto de Scielo, destacan las revistas Suma Psicológica (18) y Pensamiento Psicológico (19), de Colombia; Terapia Psicológica (20), de Chile; Anales de Psicología (16), Clínica y Salud (17) y la Revista de la Asociación Española de Neuropsiquiatría, de España; y la Revista de Neuro-Psiquiatría, (21) y Liberabit (22), de Perú. Esta última ha sido estudiada bibliométricamente (23).

Enfocándonos sólo en las revistas centradas en psiquiatría, encontramos que las que tienen los índices h5 más altos son: Revista de Psiquiatría y Salud Mental (España) (h5=21), Revista Colombiana de Psiquiatría (h5=20), Actas Españolas de Psiquiatría (h5=16), Revista de Neuro-Psiquiatría (Perú) (h5=15) y Salud Mental (México) (h5=15). Este mismo indicador, correspondiente al periodo 2014-2018, encuentra casi a las mismas revistas de psiquiatría mencionadas arriba como las de mayor impacto pero en orden algo diferente: 1) Salud Mental, 2) Actas Españolas de Psiquiatría, 3) Revista Colombiana de Psiquiatría, 4) Revista de Psiquiatría y Salud Mental y 5) Revista de Neuro-Psiquiatría con el mismo h5 que la Revista de la Asociación Española de Neuropsiquiatría (5). Estos cambios en la posición de las publicaciones se deben, por un lado, a un aumento del h5 de la Revista de Psiquiatría y Salud Mental, de la Revista Colombiana de Psiquiatría y de la Revista de Neuro-Psiquiatría; y por otro, a una leve disminución del h5 de la revista Salud Mental de México. Una explicación de esto último podría ser la falta de indexación de Salud Mental a PubMed.

Aunque el objetivo del artículo es evaluar sólo las revistas publicadas en castellano, se encontraron revistas de Brasil que además de publicar en portugués lo hacen en español e inglés; por otro lado, Salud Mental (México) actualmente publica principalmente en idioma inglés (24).
En cuanto al Perú, las revistas identificadas fueron las siguientes: Revista de Psicología (Pontificia Universidad Católica del Perú) (25), Revista de NeuroPsiquiatría (Universidad Peruana Cayetano Heredia) (26), Liberabit (Universidad San Martín de Porres) (22), Persona (Universidad de Lima) (27) y Revista de Investigación en Psicología (Universidad Nacional Mayor de San Marcos) (28). Todas estas revistas son auspiciadas por alguna universidad. La Revista de Psicología es la única publicación peruana sobre salud mental incluída en Scopus.

Los países de habla hispana con mayor número de revistas de salud mental (España, Colombia, México y Chile) también son los que tienen mayor producción científica y mayor impacto de sus publicaciones en este campo (5).

En general, las revistas con mejores indicadores de impacto son también las que tienen las tasas de aceptación de artículos más bajas (29), por lo que es importante tener en cuenta a las diversas revistas con sus diferentes indicadores de impacto al momento de escoger dónde enviar el manuscrito, de modo que se eviten rechazos repetidos que generarían un retraso importante en la publicación o el abandono del deseo de publicar por parte de los autores.

Entre las limitaciones del presente trabajo tenemos que no se revisó la base de datos de Pubmed ni se analizaron los indicadores de Web of Science (30). Por otro lado no se consideró información referente al efecto que pudiera tener sobre el impacto de la revista si ésta tiene versión impresa o es exclusivamente digital, si es auspiciada por alguna universidad, o si se publica exclusivamente en español, inglés o ambos idiomas.

En conclusión, las revistas sobre psiquiatría y salud mental que aceptan publicaciones en español que están mejor posicionadas en cuanto a su impacto son de España y entre ellas destaca Psicothema. Los resultados de este estudio podrían ayudar a los autores, que trabajan en salud mental y que desean publicar sus artículos en idioma español, a escoger entre una diversidad de revistas científicas. Asimismo, serían de utilidad a los editores y cuerpos editoriales de las revistas de psiquiatría y salud mental para los planes de mejoramiento de sus publicaciones y sus indicadores de impacto. 


\section{Correspondencia:}

Dr. Johann M. Vega-Dienstmaier

Correo electrónico: johann.vega.d@upch.pe, johannvega@yahoo.com

Conflictos de interés: El autor es editor asociado de la Revista de Neuro-Psiquiatría.

Financiamiento: Por el propio autor.

\section{REFERENCIAS BIBLIOGRÁFICAS}

1. Glänzel W, Moed HF. Journal impact measures in bibliometric research. Scientometrics. 2002;53: 171193. DOI:10.1023/A:1014848323806

2. Roldan-Valadez E, Salazar-Ruiz SY, Ibarra-Contreras R, Rios C. Current concepts on bibliometrics: a brief review about impact factor, Eigenfactor score, CiteScore, SCImago Journal Rank, SourceNormalised Impact per Paper, H-index, and alternative metrics. Ir J Med Sci. 2019;188: 939-951. doi: 10.1007/s11845-018-1936-5

3. Valle R, Rivera-Encinas MT, Stucchi-Portocarrero S. Producción, impactoy colaboración en investigaciones peruanas en psiquiatría y salud mental. Acta Med Peru. 2020;37:285-293.Doi:10.35663/amp.2020.372.1072

4. Luna-Solís Y. Producción científica en salud mental en Perú: Reto en tiempos de reforma de salud. Acta Med Peru. 2015;32: 36-40.

5. Vega-Dienstmaier JM. Publicaciones científicas peruanas sobre salud mental. Rev Neuropsiquiatr. 2019;82: 163-165.

6. SciELO. Revistas. Sao Paulo: SciELO; 2021.(Citado el 3 de octubre de 2021). Disponible en: https://www. scielo.org/es/revistas/listar-por-orden-alfabetico

7. Google Académico. Publicaciones principales. Google; 2021.(Citado el 3 de octubre de 2021). Disponible en: https://scholar.google.es/ citations?view_op=top_venues\&hl=es

8. Scopus. Sources: Improved Citescore. Elsevier; 2021. (Citado el 3 de octubre de 2021). Disponible en: https://www.scopus.com/sources

9. Google Académico. Google Scholar Metrics. Google; 2021. (Citado el 3 de octubre de 2021). Disponible en: https://scholar.google.es/intl/es/scholar/metrics. html

10. Miyahira J. Criterios de calidad de las revistas científicas. Rev Med Hered. 2008;19: 1-4.

11. Psicothema; Oviedo: Facultad de Psicología, Universidad de Oviedo; 2021. (Citado el 9 de octubre de 2021). Disponible en: http://www.psicothema. $\mathrm{com} /$

12. Rodríguez SM, Moreno AS. Análisis bibliométrico de la revista Psicothema (1989-1997). Psicothema. 1998;10: 23-27.
13. Buela-Casal G, Medina A, Viedma MI, Godoy V, Lozano S, Torres G. Factor de impacto de tres revistas españolas de Psicología. Psicothema. 2004; 16: 680688.

14. Zych I, Buela-Casal G. Índice de internacionalidad de las revistas iberoamericanas de psicología incluidas en la Web of Science. Revista Mexicana de Psicología. 2007;24: 15-22.

15. Revista de Psiquiatría y Salud Mental. Sociedad Española de Psiquiatría; Sociedad Española de Psiquiatría Biológica; Elsevier; 2021. (Citado el 9 de octubre de 2021). Disponible en: https://www.elsevier. es/es-revista-revista-psiquiatria-salud-mental--286

16. Universidad de Murcia. Anales de Psicología. Murcia: Universidad de Murcia; 2021. (Citado el 9 de octubre de 2021). Disponible en: https://revistas.um.es/ analesps/

17. Colegio Oficial de la Psicología de Madrid. Clínica y Salud. Madrid: Colegio Oficial de la Psicología de Madrid; 2021. (Citado el 9 de octubre de 2021). Disponible en: https://scielo.isciii.es/scielo. php?script=sci_serial\&pid=1130-5274

18. Suma Psicológica. Bogota: Fundación Universitaria Konrad Lorenz; 2021.(Citado el 9 de octubre de 2021). Disponible en: http://www. scielo.org.co/scielo.php?script=sci_serial\&pid= 0121-4381\&lng=es\&nrm=iso

19. Pensamiento Psicológico. Cali: Pontificia Universidad Javeriana; 2021. (Citado el 9 de octubre de 2021). Disponible en: http://www.scielo.org.co/ scielo.php?script $=$ sci_serial\&pid $=1657-8961 \&$ lng=es

20. Terapia Psicológica. Santiago: Sociedad Chilena de Psicología Clínica; 2021.(Citado el 9 de octubre de 2021). Disponible en: https://www.scielo.cl/scielo. php?script $=$ sci_serial\&pid $=0718-4808$

21. Revista de Neuro-Psiquiatría. Lima: Facultad de Medicina, Universidad Peruana Cayetano Heredia; 2021.(Citado el 9 de octubre de 2021). Disponible en: Available: http://www.scielo.org.pe/scielo. php?pid=0034-8597\&script=sci_serial

22. Liberabit. Lima: Escuela Profesional de Psicología, Universidad San Martin de Porres; 2021. Citado el 12 de octubre de 2021). Disponible en: http://www. revistaliberabit.com/index.php/Liberabit

23. Romero J. Estudio bibliométrico de la revista Liberabit en el período comprendido entre 19952013. Liberabit. 2014;20: 369-372.

24. Salud Mental. Ciudad de México: Instituto Nacional de Psiquiatría Ramón de la Fuente Muñiz; 2021 (Citado el 12 de octubre de 2021). Disponible en: http://www.scielo.org.mx/scielo.php?script=sci serial\&pid $=0185-3325$

25. Revista de Psicología. Lima: Pontificia Universidad Católica del Perú ; 2021.(Citado el 12 de octubre de 2021). Disponible en: https://revistas.pucp.edu.pe/ 
Impacto de las revistas de psiquiatría y salud mental editadas en español.

index.php/psicologia

26. Revista de Neuro-Psiquiatría Lima: Facultad de Medicina, Universidad Peruana Cayetano Heredia; 2021. (Citado el 12 de octubre de 2021). Disponible en: https://revistas.upch.edu.pe/index.php/RNP

27. Persona. Lima: Universidad de Lima: 2021. (Citado el 12 de octubre de 2021). Disponible en: https:// revistas.ulima.edu.pe/index.php/Persona

28. Revista de Investigación en Psicología. Lima: Universidad Nacional Mayor de San Marcos; 2021. (Citado el 12 de octubre de 2021). Disponible en: https://revistasinvestigacion.unmsm.edu.pe/index. $\mathrm{php} / \mathrm{psico}$

29. Sugimoto CR, Larivière V, Ni C, Cronin B. Journal acceptance rates: A cross-disciplinary analysis of variability and relationships with journal measures. J Informetr. 2013;7: 897-906.

30. Scimago Lab. Scimago Journal \& Country Rank; 2021. (Citado el 12 de octubre de 2021). Disponible en: https://www.scimagojr.com/journalrank.php

Recibido: 18/11/2021

Aceptado: 27/12/2021 